\title{
Tumor location impacts immune response in mouse models of colon cancer
}

\author{
Xianda Zhao ${ }^{1}$, Lihua Li ${ }^{1}$, Timothy K. Starr ${ }^{2}$ and Subbaya Subramanian ${ }^{1,3}$ \\ ${ }^{1}$ Department of Surgery, University of Minnesota Medical School, Minneapolis, MN, USA \\ ${ }^{2}$ Department of Obstetrics and Gynecology and Women's Health, University of Minnesota Medical School, Minneapolis, MN, USA \\ ${ }^{3}$ Masonic Cancer Center, University of Minnesota, Minneapolis, MN, USA \\ Correspondence to: Subbaya Subramanian, email: subree@umn.edu \\ Keywords: colorectal cancer, immune profiles, immunotherapy, mouse models of cancer, orthotopic models \\ Received: March 21, $2017 \quad$ Accepted: May 23, $2017 \quad$ Published: June 09, 2017 \\ Copyright: Zhao et al. This is an open-access article distributed under the terms of the Creative Commons Attribution License 3.0 \\ (CC BY 3.0), which permits unrestricted use, distribution, and reproduction in any medium, provided the original author and source \\ are credited.
}

\section{ABSTRACT}

Existing preclinical models of human colorectal cancer (CRC) that rely on syngeneic subcutaneous grafts are problematic, because of increasing evidence that the immune microenvironment in subcutaneous tissue is significantly different from the gastrointestinal tract. Similarly, existing orthotopic models that use a laparotomy for establishing grafts are also problematic, because the surgical procedure results in extensive inflammation, thereby creating a nonphysiologic tumor microenvironment. To facilitate the bench-to-bedside translation of CRC immunotherapy strategies, we developed a novel orthotopic model in mice that uses endoscopy-guided microinjection of syngeneic cancer cells. When we compared immune system infiltration, we found that tumors in the subcutaneous model had fewer $\mathbf{T}$ cells, B cells, and natural killer (NK) cells, but more immunosuppressive myeloid cells; in contrast, tumors in our orthotopic model had a higher number of tumor-infiltrating T cells, B cells, and NK cells, with fewer immunosuppressive myeloid cells. The number of immune-stimulating cytokines, such as interleukin (IL)-2, IL-6, interferon (IFN)-gamma, and granzyme B, was also higher in tumors in our model, as compared with the subcutaneous model. Those differences resulted in heightened sensitivity to immune checkpoint blockade therapy in our endoscopy-guided orthotopic CRC model. Our study indicates that tumor location affects immune response in CRC mouse models; choosing the appropriate preclinical model is important when testing immunotherapy in CRC.

\section{INTRODUCTION}

Tumor development in humans is regulated by tumor-specific adaptive immune responses [1]. Recently developed therapies that enhance the immune response, such as chimeric antigen receptor (CAR) $\mathrm{T}$ cells and immune checkpoint blockade therapies (ICBTs), have resulted in remarkable outcomes in certain cancers $[2,3]$. For example, checkpoint blockade antibodies targeting PD1, PDL1, or CTLA4 have resulted in significantly improved survival in patients with advanced drug-resistant melanoma, lung cancer, and renal cancer [4-6]. However, in patients with colorectal cancer (CRC), which ranks among the most common malignancies in the United
States, the efficacy of these therapies was much less remarkable [7].

Preclinical CRC immunotherapy studies have largely depended on syngeneic subcutaneous tumor models $[8,9]$. A recent study has shown that tumor location determines tissue-specific recruitment of tumorassociated macrophages in melanoma model [10]. Therefore, the classic subcutaneous models may not mimic the immune tumor microenvironment of actual human CRC and may be a major barrier in efforts to translate findings on immunotherapy from the laboratory to the clinic [11]. Moreover, two immune-infiltrated subtypes of CRC have been seen in human patients: the well-infiltrated subtype and the poorly infiltrated subtype 
$[12,13]$. However, the current subcutaneous CRC models are poorly infiltrated [14]. Therefore, it is critical to establish a preclinical model that recapitulate primary tumor microenvironment and mimic immune wellinfiltrated human CRC features, especially their baseline immune response. Such a model would be complementary to the classic subcutaneous models and would facilitate investigation of the mechanisms of ICBT resistance in various clinical settings [15].

Orthotopic tumor models have several advantages over chemically- or genetically-induced tumor models. In general, orthotopic transplants are easier and faster to establish, and they are located within a physiological and microenvironment comparable to that of human diseases [16]. In CRC, orthotopic tumor models have usually required a laparotomy, which can cause a strong inflammatory response, which is a potential confounding factor of experimental outcomes, making it unsuitable for immunotherapy studies [17]. We have established a novel orthotopic CRC model that uses endoscopyguided microinjection to establish orthotopic tumors in the colon wall in mice [18]. Our minimally invasive CRC model does not provoke an inflammatory response and is particularly suitable for immunotherapy studies. In this study, we investigated the key characteristics of our model, including immune infiltration and responses to ICBT. We compared orthotopic tumors with subcutaneous tumors established from the same CRC cell lines and demonstrate a significant difference in immune response. These findings highlight that tumor location influences immune responses in CRC animal models and the importance of model selection in preclinical immunotherapy studies.

\section{RESULTS}

\section{Establishment of orthotopic colorectal tumors in mouse}

To establish a standard procedure of tumor cell implantation in the mouse colon wall, we tested different anesthesia options. We found that colon spasms and colonic secretion, in response to endoscopic examination or needle puncture, were the most common issues leading to implantation failure. The frequency and degree of colon spasms and colonic secretion were higher in mice anesthetized with Avertin; those issues were controlled when we administered a combination of ketamine, xylazine, and atropine. During the process of tumor cell implantation, a positive lifting of the colonic mucosa at the implantation sites indicated successful cell inoculation (Supplementary Video 1). We have achieved $80 \%$ success rate in implanting orthotopic mouse CRC tumors.

When we used endoscopy to monitor tumor growth, we saw abnormal protrusions in the colon lumen around 1 week after injection in some mice (Figure 1A, i-ii); subsequently, rapid tumor growth induced colonic obstruction (Figure 1A, iii). In other mice, as tumors invaded the submucosal layer and expanded toward the serosa, we could not detect tumor growth by bright-light endoscopy; however, we did see stiffness, brittleness, and heavy bleeding in the mucosa (Figure 1A, iv-vi). Histologic analysis indicated that orthotopic tumors were growing in the submucosa layer and invading the muscularis layer (Figure 1A, vii). We detected orthotopic tumors around 3 weeks after implantation of $10^{5}$ CT26Luc cells (Figure 1B) by IVIS. A direct correlation between tumor volume and the number of injected tumor cells can be found in our orthotopic model (Figure 1C).

\section{Fewer cells are required to establish subcutaneous models compared to orthotopic models}

In athymic nude mice, orthotopic injection of $10^{3}$ HT29 cells could induce tumor formation at 4 weeks in the subcutaneous model, but not in orthotopic model (Figure 1D). Orthotopic injection of $10^{5}$ HT29 cells was sufficient to induce tumor formation and invasion at 4 weeks in the orthotopic model (Supplementary Figure 1A). Similar results were observed in our two syngeneic orthotopic CRC models. For example, injection of $10^{4} \mathrm{CT} 26$ cells induced tumor formation at 4 weeks in subcutaneous tissue, but not in the colon wall in BALB/c mice (Figure 1E; results confirmed by autopsy, data not shown) and injection of $10^{5} \mathrm{MC} 38$ cells induced tumor formation at 4 weeks in subcutaneous tissue, but not in the colon wall in C57BL/6 mice (Figure 1F). Taken together, our data indicate that more cells are required to initiate tumorigenesis in the orthotopic model compared to subcutaneous model.

\section{Orthotopic tumors have more adaptive immune- cell infiltration than subcutaneous tumors}

Multiple studies have demonstrated that immune cell infiltration in CRC is highly variable between patients and increased infiltration of immune cells was associated with a better outcomes [19]. Immunostaining with T-cell markers (CD3 and CD8) in human CRC samples, we also observed two CRC subtypes, based on T-cell infiltration: well-infiltrated tumors (Figure 2A, i-iii) and poorlyinfiltrated tumors (Figure 2A, iv). In samples of normal colon from $\mathrm{BALB} / \mathrm{c}$ mice, the microenvironment that orthotopic tumors grew in, we found very few $\mathrm{T}$ cells except in the Peyer patches (Figure 2B). This observation may be reflective of the lacks of antigen exposure in these mice and their controlled housing environment [20]. In the orthotopic tumor tissue, we found $\mathrm{T}$ cells in both the margins (Figure 2C, i) and the central parts (Figure $2 \mathrm{C}$, ii) of tumors; we saw both $\mathrm{CD}^{+}$(Figure $2 \mathrm{C}$, iii) and $\mathrm{CD} 8^{+}$cells (Figure $2 \mathrm{C}$, iv). In contrast, in subcutaneous tumors established by the same cell line, we found a very small number of $\mathrm{T}$ cells (Figure 2D). Flow cytometry 

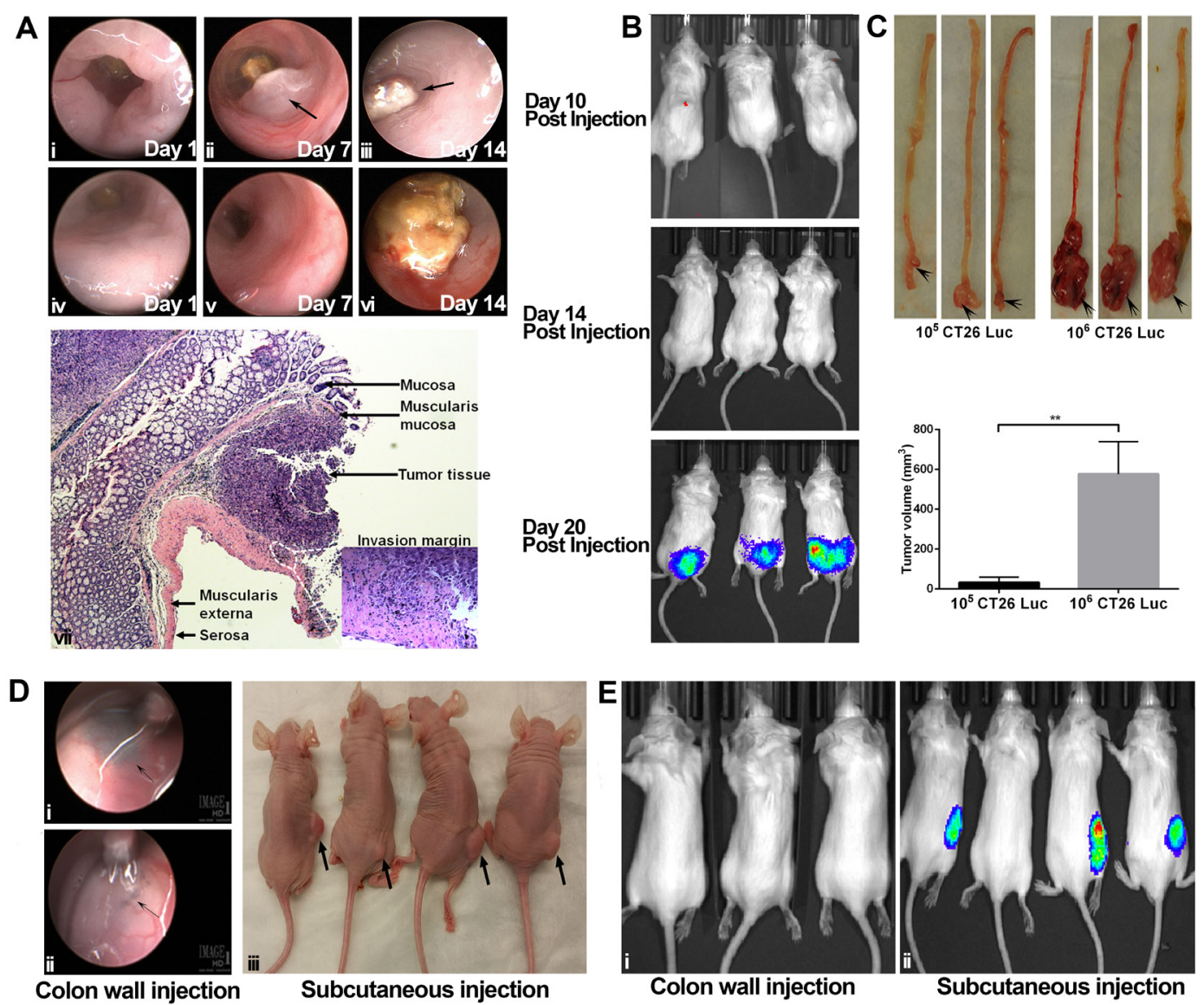

F

14 days post injection

28 days post injection
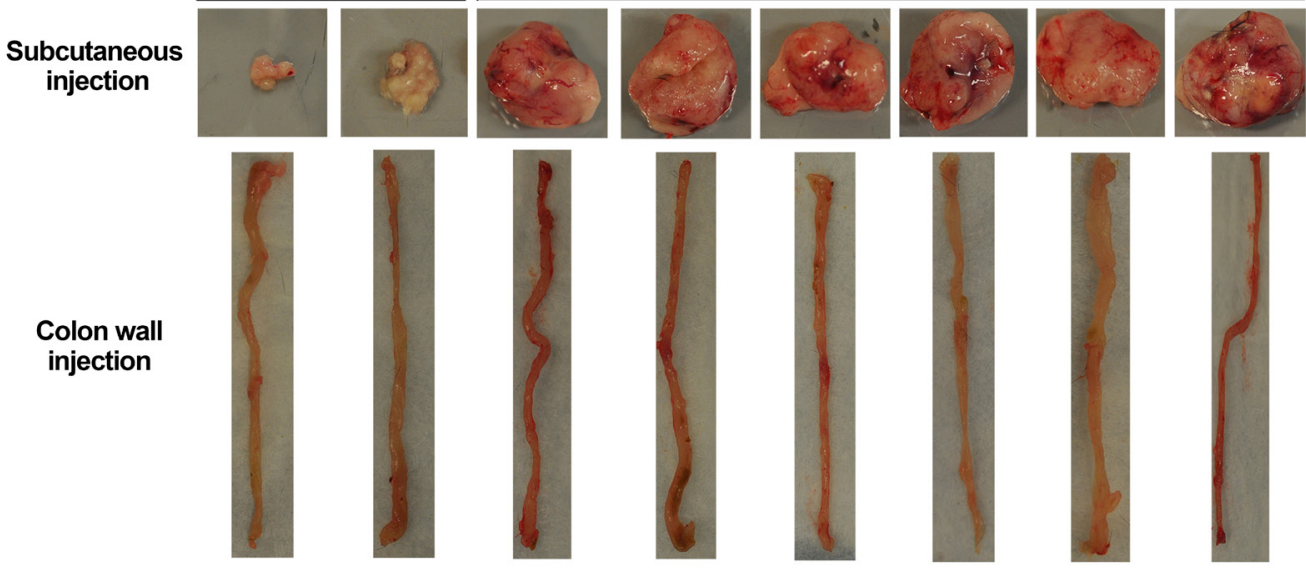

Figure 1: Tumorigenesis in orthotopic and subcutaneous models was distinct. Orthotopic tumors were established by endoscopy-guide microinjection in the colon wall of BABL/c mice. In some mice, tumors and tumor-caused colon obstruction can be seen directly in the colon lumen (A, i-iii). In other mice, abnormal movement, stiffness, and bleeding can be seen in the colon (A, iv-vi). H\&E staining showed tumor tissue in the submucosa layer. The invasion margin can be seen in the muscularis externa (A, vii). An in vivo imaging system can be used for monitoring in our orthotopic model (B). Injection of different numbers of CT26 cells $\left(10^{5}\right.$ and $\left.10^{6}\right)$ led to a significance difference in tumor volume at autopsy (C). At 4 weeks after injection of $10^{3}$ HT29 tumor cells in either the colon wall or the subcutaneous connective tissue of athymic nude mice $(\mathrm{n}=4$ in each model), tumorigenesis occurred only in the subcutaneous model (D). Arrows indicate the representative injection sites in the colon wall, but no tumors formed (D, i-ii). At 4 weeks after injection of $10^{4}$ CT26 cells in either the colon wall $(\mathbf{E}, \mathbf{i} ; n=3)$ or the subcutaneous connective tissue $(\mathbf{E}, \mathbf{i i} ; \mathbf{n}=4)$ of BALB/c mice, subcutaneous tumors formed (E, i); however, no orthotopic tumors formed (E, ii). Autopsy confirmed the results of imaging (data not shown) (E). Injection of $10^{5} \mathrm{MC} 38$ did not induce tumor formation in the colon wall $(\mathrm{n}=8)$ of C57/B6 mice, whereas it did cause subcutaneous tumor formation $(\mathrm{n}=8)(\mathbf{F})$. H\&E: hematoxylin and eosin. ${ }^{* *} P<0.01$. 
analysis showed the same trends of T-cell infiltration in both models. We then determined the proportion of $\mathrm{CD}^{+}$ $\mathrm{T}$ cells in these two models and found no differences. Since B cells are important in antigen presentation and adaptive immunity. We checked B-cell number in both these models and found the number of tumor-infiltrating B cells was higher in orthotopic tumors than in subcutaneous tumors (Figure 2E). RT-qPCR analysis showed expression of chemokines related to T-cell migration was higher in orthotopic tumors than in subcutaneous tumors (Figure 2F).

\section{NK cells increased and myeloid-derived suppressive cells are decreased in orthotopic tumors}

Innate immune cells are also critical in regulating antitumor immune responses. Therefore we compared the innate immune profiles between these two models. We observed high levels of NKp46 $6^{+}$cells in human normal colon tissues (Figure 3Ai). However, in CRC patient samples, we observed differential levels of $\mathrm{NKp}^{+} 6^{+}$ cells. (Figure 3Aii, 3Aiii). In mice tissue samples, we found more $\mathrm{NKp} 6^{+}$cells in orthotopic tumors than in subcutaneous tumors (Figure 3B, 3C). RT-qPCR analysis of transcript levels of genes that encode cytokines or chemokines related to natural killer (NK) cell functions, we found higher expression of IL12, IL15, and IL18 in orthotopic tumors than subcutaneous tumors (Figure 3D). Flow cytometry analyses showed no difference in the number of dendritic cells (DCs) and macrophages between the two models (Figure 3E). We have carried out staining for $\mathrm{CD}^{2} 0^{+}$(M1 subtype) and $\mathrm{CD} 206^{+}$(M2 subtype) in both of our CRC models. Our data showed no significant difference in the proportion of the M1 and M2 subtypes (Supplementary Figure 2). However, we found more $\mathrm{CD}_{11} \mathrm{~b}^{+}, \mathrm{CD} 11 \mathrm{c}^{-}$, and $\mathrm{Ly}_{6 \mathrm{C}^{+}}$or $\mathrm{Ly}_{6 \mathrm{G}^{+}}$myeloid-derived suppressor cells (MDSCs) in subcutaneous tumors than orthotopic tumors (Figure 3F).

\section{Overall antitumor immune response increased in orthotopic tumors}

Our analysis indicated a more robust immune cell presence in the orthotopic model. Further, we determined the overall inflammatory and antitumor immune response intensity by measuring the concentrations of IL6, IL2, IFN $\gamma$ and granzyme B. We found that CT26 cells and normal colon tissues did not express IL6, IL2, IFN $\gamma$ and granzyme B. However, compared with subcutaneous tumors, orthotopic tumors that grew in the colon microenvironment showed higher expression of all the cytokines tested (Figure 4). These findings support our observation that orthotopic tumors tend to have more antitumor immune cells and fewer immunosuppressive cells, suggesting that orthotropic tumors have a stronger overall antitumor immune response than subcutaneous tumors.

\section{Immune checkpoint expression and efficacy of ICBT differed between orthotopic and subcutaneous models}

Besides the number of immune cells, the activation status of cytotoxic immune cells is also critical in regulating antitumor immune response. The functional status of immune cells in tumors was determined by measuring the expression levels of immune checkpoints and T-cell activation markers. We generated orthotopic and subcutaneous CRC models by injecting same number $\left(10^{5}\right)$ of cells. In subcutaneous tumors, we observed higher levels of immune checkpoints such as CTLA4 and PD1 on $\mathrm{T}$ cells compared to orthotopic tumors (Figure 5A). Further, PDL1 expression was also higher in subcutaneous tumors than in orthotopic tumors (Figure 5A). However, we observed no significant differences in expression of T-cell activation markers such as CD62L, CD44, and IFN $\gamma$ in tumor-infiltrating $\mathrm{T}$ cells in both tumor models (Figure 5B). We further investigated whether these two tumor models with different immune profiles show varying response to immune checkpoint blockade therapy (ICBT). Towards this, mice were either treated with IgG or anti-PD1 plus anti-CTLA4 in six doses for three weeks (Figure 5C). This treatment regime induced response in both orthotopic and subcutaneous tumors (Figure 5D, 5E, and $5 \mathrm{~F}$ ). After six doses of treatment, we did not observe any tumor growth in the orthotopic model (Figure 5D). However, in subcutaneous model, although the tumors responded to ICBT (Figure 5E and 5F, Supplementary Figure 3), residual tumors were still observed. To determine tumor vascularization that may have potential confounding effect on ICBT treatment, we stained CD31 in two of our CRC models. Our staining patterns suggest that no obvious difference of capillary density in these two models (Supplementary Figure 4).

\section{DISCUSSION}

Studies in mice are frequently used to provide the rationale for testing anti-tumor therapies in phase 1 clinical trials [21]. The traditional approach, using human xenografts in immuno-compromised mice, is not amenable to testing immunomodulatory anti-tumor agents due to the absence of a physiological immune system. The mouse models of tumors, including human xenograft tumors and syngeneic tumors, were suitable for preclinical studies that tested cytotoxic drugs [21]. However, immunotherapy has a different mechanism for eliminating tumors: it kills tumor cells by enhancing or rebuilding the antitumor immune response in patients (rather than by directly killing tumor cells)[1]. The efficacy of immunotherapy, especially 
ICBT largely depends on the immune microenvironment of tumors [22, 23].

To maximize opportunities to translate novel immunotherapy strategies from preclinical studies to clinical application, CRC mouse models are needed that can mimic physiologically relevant microenvironment
[11]. In this study, we analyzed two immune-competent models using murine CRC cell lines (CT26 and MC38) in their matched immune-competent hosts (BALB/c and C56BL/6). We demonstrated that these models can be used to test the efficacy of checkpoint blockade therapy, antiCTLA4 and anti-PD1. Furthermore, we demonstrated that
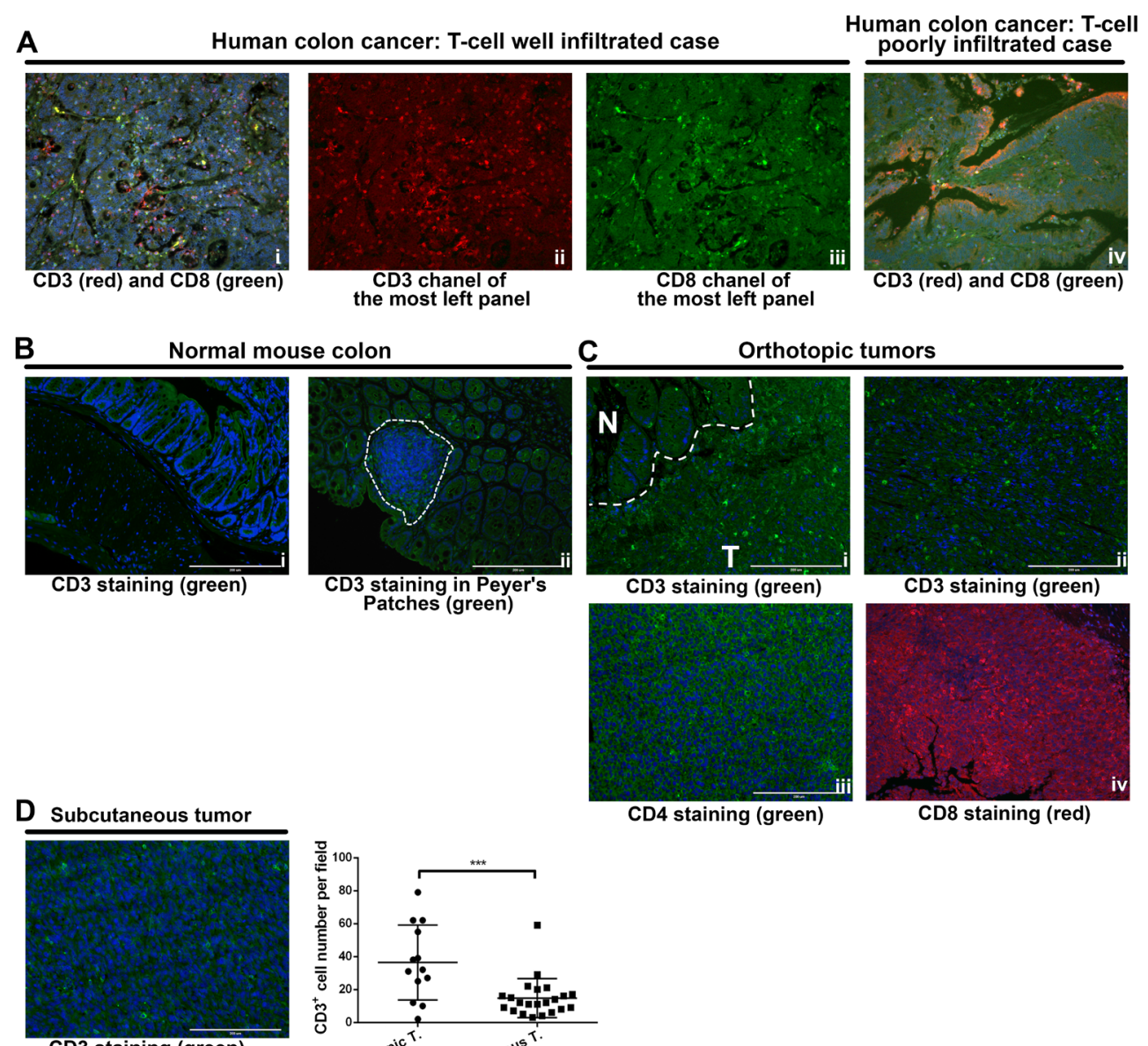
Patches (green)

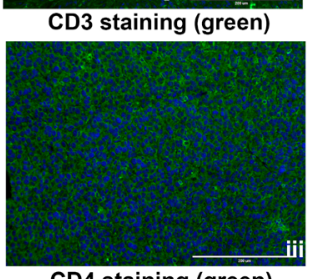

CD4 staining (green)

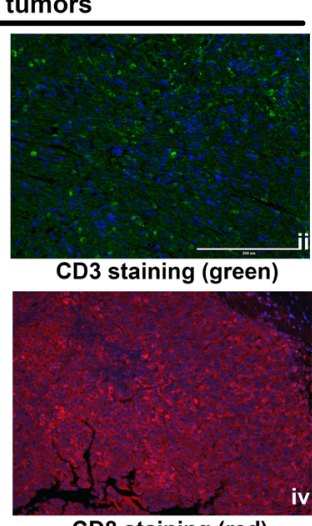

CD8 staining (red)

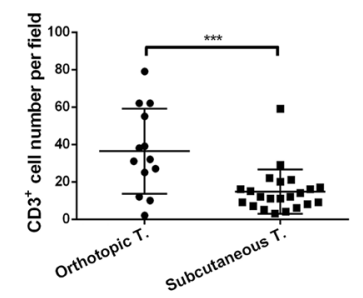

E
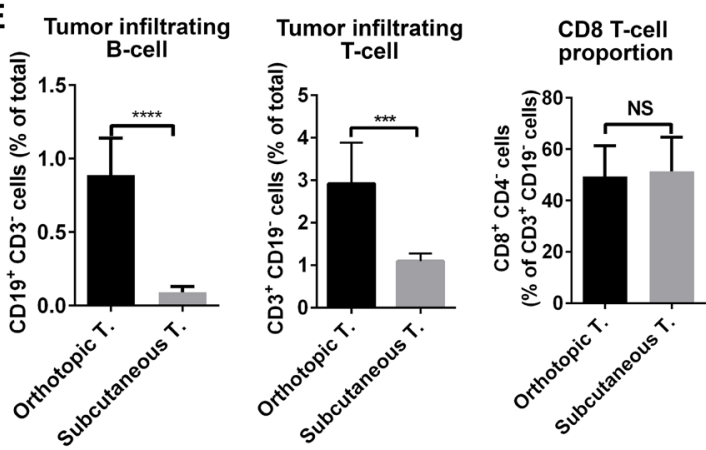

$\mathbf{F}$

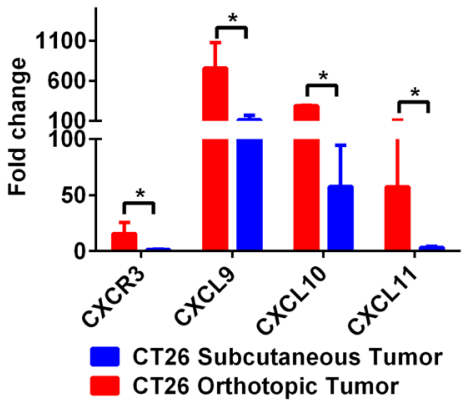

Figure 2: Adaptive immune cell infiltration was higher in orthotopic tumors than in subcutaneous tumors. In human CRC samples, both well-infiltrated (A, i-iii) and poorly-infiltrated (A, iv) tumors were found. In normal BALB/c mouse colon, CD3 ${ }^{+}$cells were usually seen in the mucosa lymph tissues $(\mathbf{B})$. In orthotopic tumors, $\mathrm{CD}^{+}$cells were seen in the tumor margins $(\mathbf{C}$, i) and in the central parts of the tumor $\left(\mathbf{C}\right.$, ii). Both $\mathrm{CD} 4^{+}$and $\mathrm{CD} 8^{+}$cells were seen in orthotopic tumors $\left(\mathbf{C}\right.$, iii-iv). In subcutaneous tumors, $\mathrm{CD} 3^{+}$cells were also detected, but not as many as in orthotopic tumors (D). Flow cytometry analysis indicated more B cells and more T cells in orthotopic tumors than in subcutaneous tumors. The proportion of $\mathrm{CD} 8^{+} \mathrm{T}$ cells was the same in the two models (E). In orthotopic tumors, mRNA expression of CXCR3, CXCL9, CXCL10, and CXCL11 was higher. Expression level was presented as fold change, refers to the lowest expression (F). N: normal; T: tumor. ( $\%$ total refers to total number of cells in tumor tissue) $* * * P<0.001 ; * * * * P<0.0001$. 
immune profiles and response are different in an orthotopic tumor microenvironment compared to subcutaneous tumors.

In line with previous studies, we observed human CRC subtypes with immune cells well- and poorly- infiltrated tumors $[12,19]$. The immune cells poorlyinfiltrated tumors are insensitive to ICBT [24], while the well -infiltrated tumors are more sensitive to ICBT [25]. Our investigation of adaptive immune profiles of orthotopic and subcutaneous tumors in mice represents a
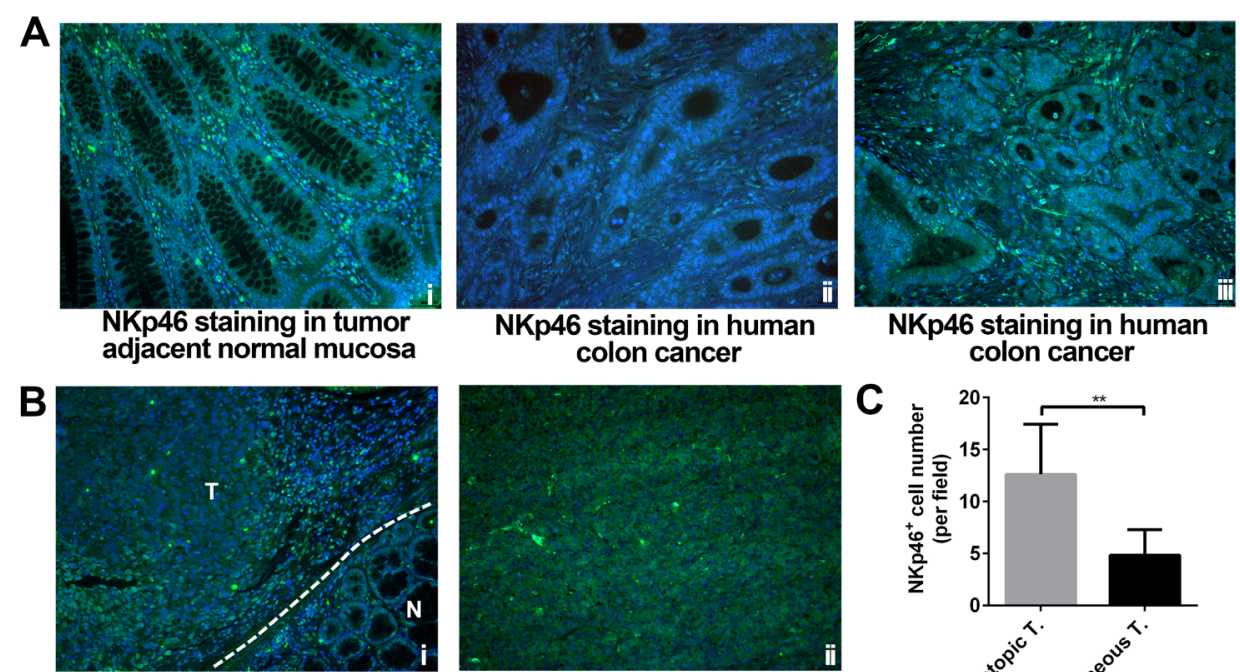

NKp46 staining in
orthotopic tumor

NKp46 staining in human colon cancer

NKp46 staining in human


\section{E Dendritic cells}
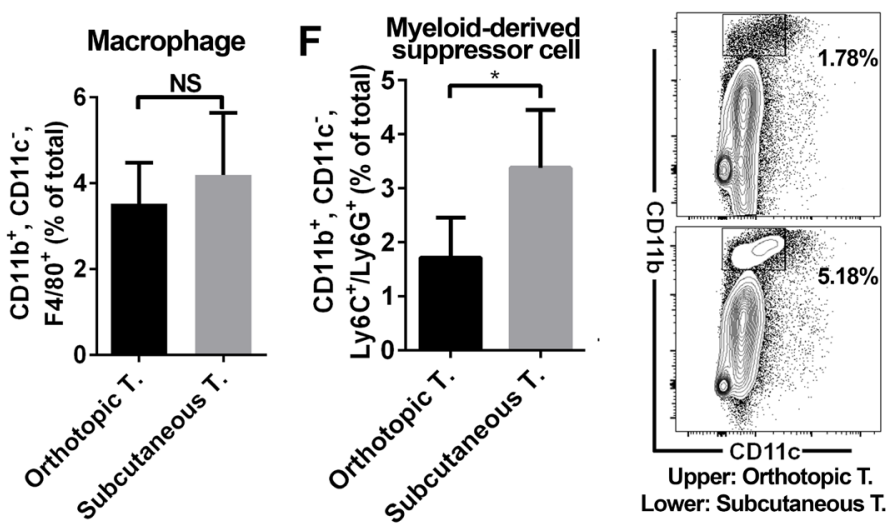

Figure 3: Innate immune cell infiltration in orthotopic and subcutaneous models was distinct. In human colon samples, $\mathrm{NKp} 46^{+}$cells were found in normal colon and in some CRC cases (A). In tumor models, NKp46 ${ }^{+}$cells were more frequent in orthotopic tumors than in subcutaneous tumors (B-C). mRNA expression of IL-12, IL-15, IL-18, and CCL5 (all related to NK cells) was higher in orthotopic tumors. Expression level was presented as fold change, refers to the lowest expression (D). Flow cytometry showed no difference in the number of DCs and macrophages in the two models (E). However, more MDSCs, important immunosuppressive cells, were found

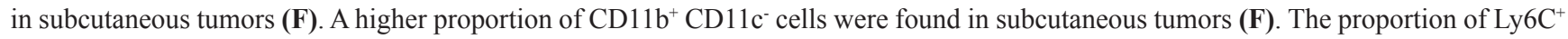
and $\mathrm{Ly}_{6 \mathrm{G}^{+}}$cells (in the $\mathrm{CD} 11 \mathrm{~b}^{+} \mathrm{CD} 11 \mathrm{c}^{-}$population) was the same in the two models (data not shown). IL: interleukin; N: normal; T: tumor. (\% total refers to total number of cells in tumor tissue) $* P<0.05$. 
key step in mimicking different clinical settings in mouse models. The two CRC tumor models produced diverse immune phenotypes in terms of $\mathrm{T}$ cells infiltration. Orthotopic tumors were better infiltrated with T cells than subcutaneous tumors, same trend was also observed with B cells. Similar results were also observed in a cecum model [26], in which higher frequency of $\mathrm{CD}^{+} \mathrm{T}$ cells were detected in Peyer's patches in response to tumor challenge. Furthermore, studies have shown that regulatory T-cell (Treg) inhibits the function of cytotoxic T-cell and selectively kills antigen presenting cells including B cells [27]. However, in our study, the proportion of Tregs among all $\mathrm{T}$ cells was similar in orthotopic and subcutaneous CRC models (data not shown). This suggests that Treg infiltration is not affected by tumor location.

Innate immune cells, depending on their differentiation and functional status, either suppressed or promoted tumor formation [28]. NK-cell is the major cell type in innate immunity that have antitumor functions [29]. Enhancing the antitumor effects of NK cells via heterodimeric bispecific single chain variable fragments (scFv) killer engagers has been very promising in preclinical models [30]. In human CRC tissue samples, we observed a subset of samples with infiltrated NK cells. In mice, orthotopic tumors had more NK-cell infiltration than subcutaneous tumors. In a caecum model, higher levels of NK cells were founded in mesenteric lymph nodes (MLNs) of tumor group compared with MLNs of control groups [26]. Notably, subcutaneous tumors had more immunosuppressive cells such as MDSCs, compared to orthotopic tumors. On the other hand, we found the same proportion of antigen-presenting cells, such as DCs and macrophages in these two tumor models. In terms of expression of IL2, IL6, IFN $\gamma$ and granzyme B, the orthotopic tumors showed relatively higher levels than subcutaneous tumors. Taken together, both adaptive and
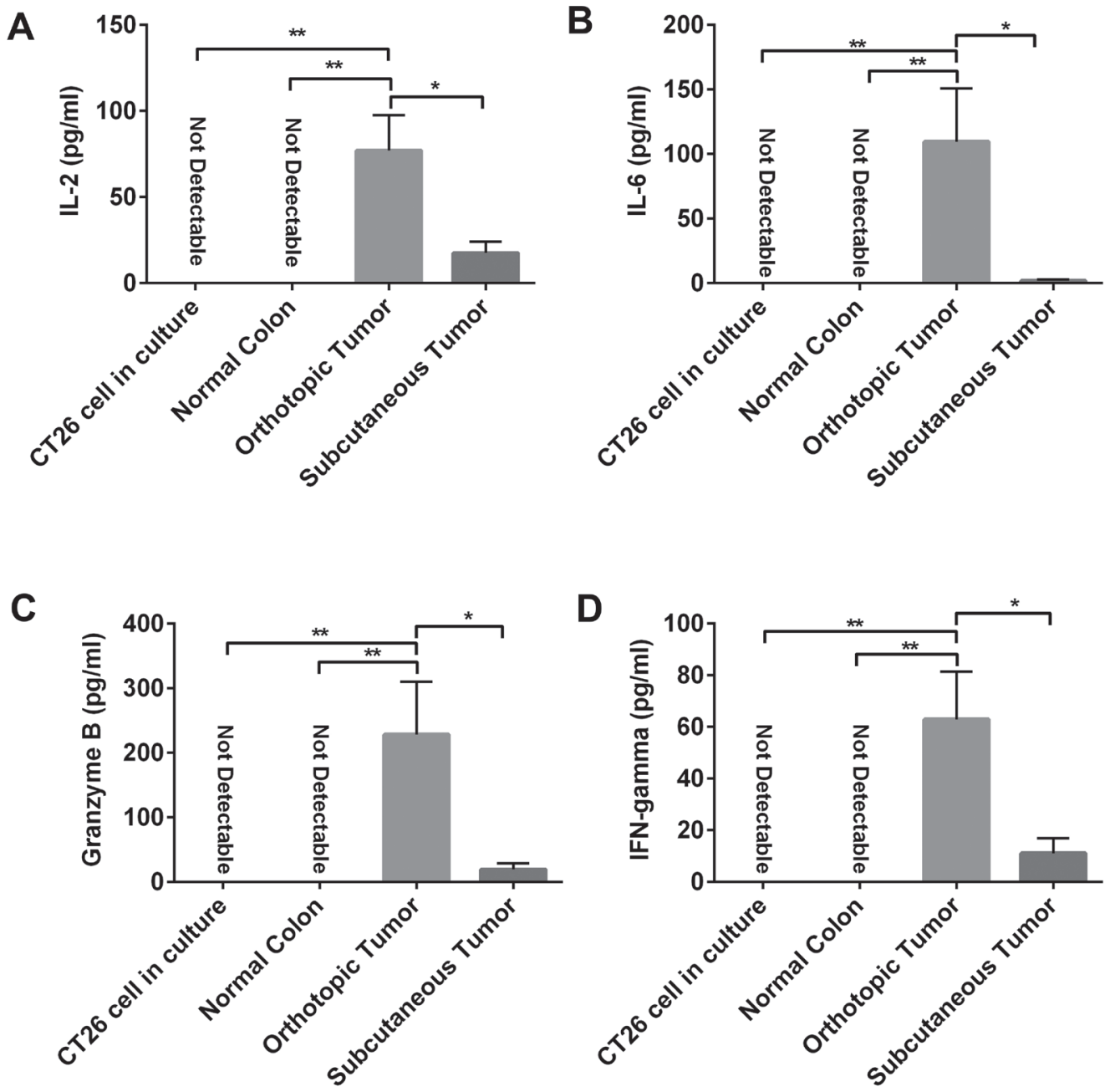

Figure 4: Expression of IL2, IL6, granzyme B, and IFN $\gamma$ varied in orthotopic and subcutaneous tumors. Using the enzyme-linked immunosorbent assay (ELISA) method, we measured the expression of some proinflammatory and cytotoxic cytokines, such as IL2 (A), IL6 (B), granzyme B (C), and IFN $\gamma$ (D). In CT26 tumor cell culture and in normal colon tissue in BALB/c mice, those cytokines could not be detected. But in both orthotopic and subcutaneous tumors in mice, we detected expression of those cytokines: expression was higher in orthotopic tumors than in subcutaneous tumors. IFN- $\gamma$ : interferon-gamma; IL: interleukin. ${ }^{*} P<0.05$; $* * P<0.01$. 
innate immune response are stronger in orthotopic model than in subcutaneous model. Similar to our observation, another study showed that tumors in the flank had poor immune response compared with tumors in the dorsal region of the foot [31]. On the other hand, the strong immune response observed in orthotopic tumors may explain why higher numbers of tumor cells are required to establish orthotopic CRC model than the subcutaneous tumors. Further studies are warranted to decipher the molecular mechanisms that regulate immune response in these models.

By comparing the two mouse models, we found that inhibitory immune checkpoint proteins, such as CTLA4, PD1, and PDL1 were expressed at lower levels
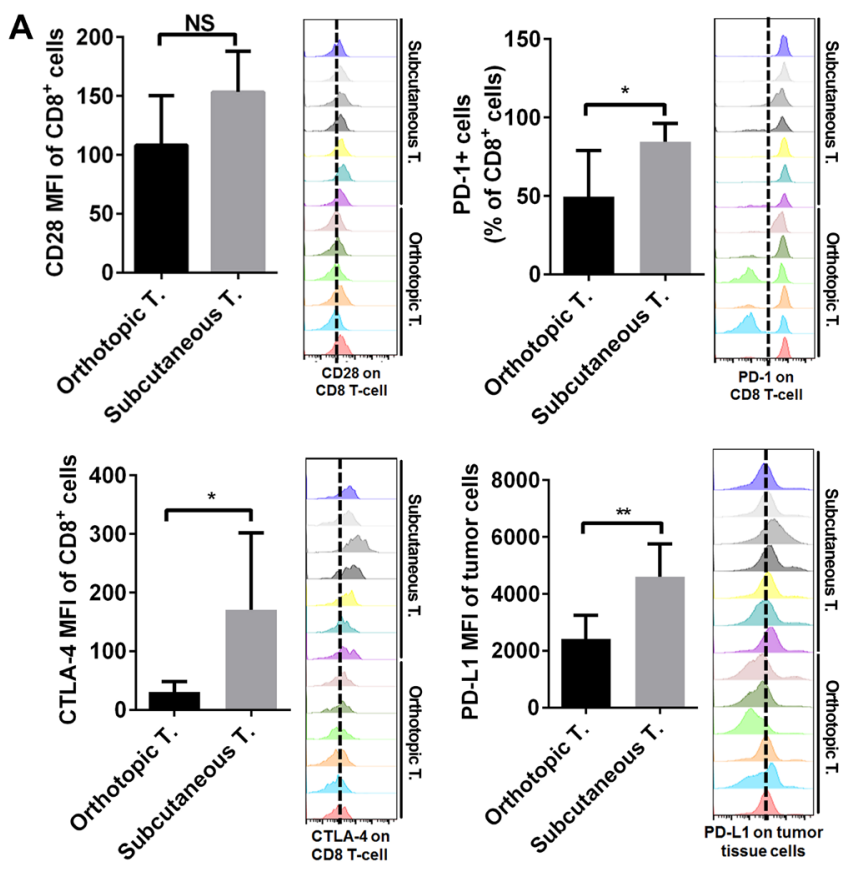

C

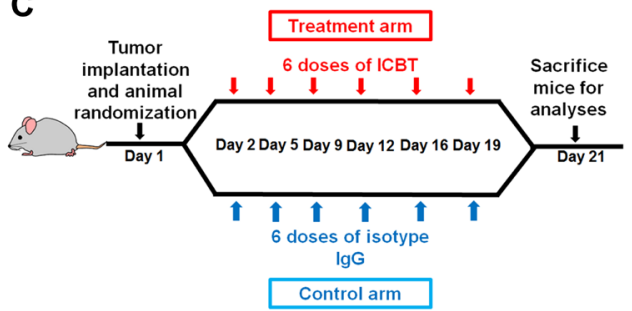

$\mathbf{F}$
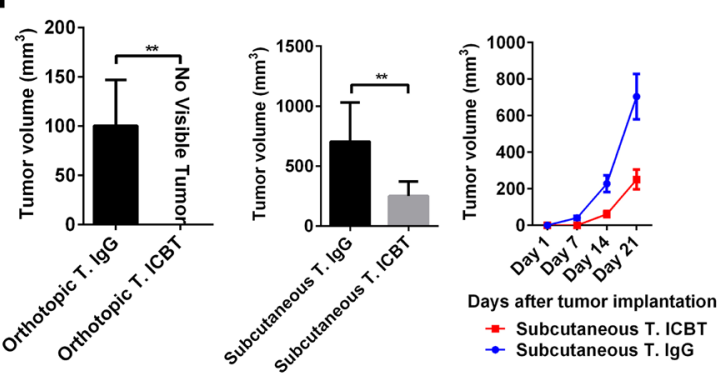

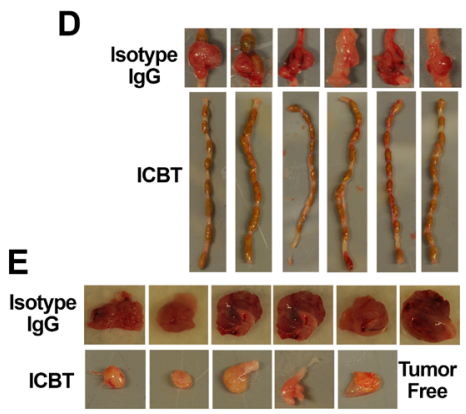

Figure 5: Immune checkpoint profile and response to ICBT differed in orthotopic and subcutaneous tumors. Expression of inhibitory immune checkpoints PD1 and CLTA4 was higher on tumor-infiltrating T cells in subcutaneous tumors (A). Expression of the stimulatory immune checkpoint CD28 was also higher on tumor-infiltrating $\mathrm{T}$ cells in subcutaneous tumors, but the difference was not statistically significant ( $\mathrm{P}=0.057)$ (A). Expression of PDL1 was higher in subcutaneous tumors (A). Expression of T-cell activation/function markers (IFN- $\gamma$, CD62L, and CD44) was the same on tumor-infiltrating T cells in subcutaneous and orthotopic tumors (B). To compare the drug sensitivity of the 2 models, we administered anti-CLTA-4 and anti-PD1 with a moderate intensity (C). At the endpoint, orthotopic tumors showed a better response; they were thoroughly blocked by ICBT, whereas, the subcutaneous tumors can only be partly controlled by ICBT (D-F). ICBT: immune checkpoint blockade therapy; IFN- $\gamma$ : interferon-gamma; T: tumor. $* P<0.05 ; * * P<0.01$. 
in orthotopic tumors compared to subcutaneous tumors. Such checkpoints are significant factors in regulating activation of adaptive immune cells, especially $\mathrm{T}$ cells $[32,33]$. We also saw a trend $(p<0.057)$ towards higher expression of the stimulatory immune checkpoint CD28 in subcutaneous tumors. These findings suggested that $\mathrm{T}$ cells in subcutaneous tumors were activated, but under strong suppression of immunosuppressive factors including immune checkpoint. These data were in line with our results that subcutaneous tumors have a weak immune response.

Response to ICBT in these two models was also investigated. Multiple ICBT protocols with large differences in treatment intensity have been reported in preclinical studies [34-36]. It also has been demonstrated that dual blockade of the PD1 and CTLA4 pathways increased the antitumor effects via enhancing immune effector cell/regulatory $\mathrm{T}$-cell ratio in an animal model [37]. Considering that the CT26 tumors were relatively sensitive to ICBT [38], we administrated immune checkpoint blockades with a moderate intensity. This treatment plan would rule out false positive or negative effects due to inappropriate therapeutic dose. We found that tumors in both models responded to ICBT. But orthotopic tumors were more sensitive: they were totally blocked by ICBT. Subcutaneous tumors responded to
ICBT, based on reduced growth; yet at the end of our study period, small subcutaneous tumors were still present. There was no difference observed in the CD31 levels between these two CRC tumor models suggesting that capillary density did not influence delivery of ICBT. Taking all data together, the subcutaneous model mimics a poor immune infiltrated and heavily immunosuppressive phenotype, whereas the orthotopic model can mimic relatively well-immune infiltrated CRC in a physiologically relevant microenvironment.

As a prerequisite for translating innovative immunotherapy strategies from bench to bedside, appropriate experimental CRC models are needed to accurately mimic different clinical settings. Our study investigated the key characteristics of a novel orthotopic CRC model, and showed significant differences between the orthotopic model and subcutaneous model in immune profiles and ICBT. Our study indicates that there remains a role for the subcutaneous model in immunotherapeutic studies, because they are easy to establish, are very stable, and they mimic a relatively sparse immune microenvironment. Our orthotopic model, on the other hand, provides another useful option, that better mimics CRC tumors with higher levels of immune infiltration in human patients (Figure 6). Taken together, our study

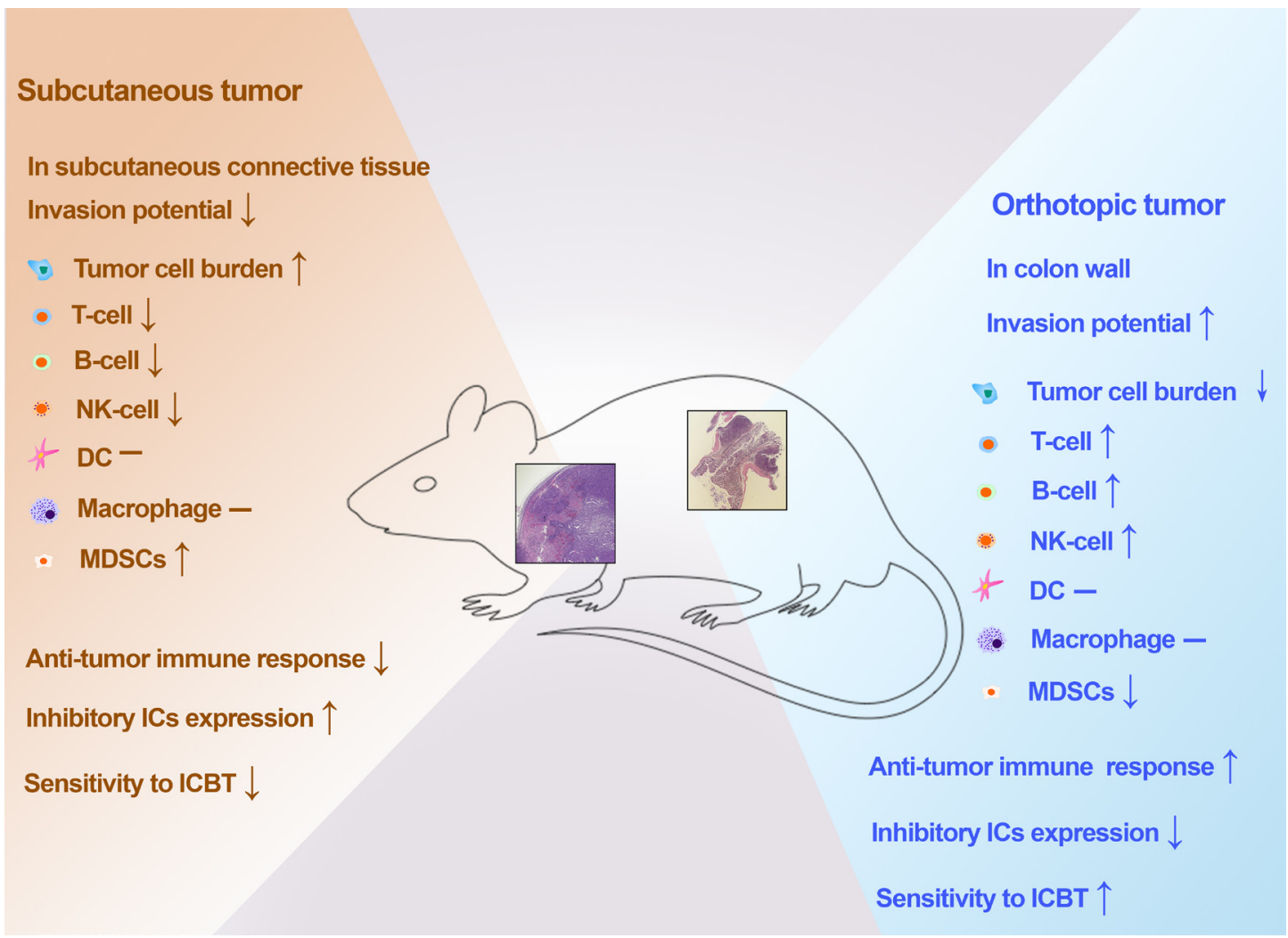

Figure 6: Summary of comparisons between the novel orthotopic CRC model and classic subcutaneous CRC model. Orthotopic tumors required a higher number of tumor cells for tumorigenesis, but showed a stronger invasion ability, antitumor immune response, and sensitivity to ICBT. ICBT: immune checkpoint blockade therapy. 
identifies the influence of tumor location on immune response in CRC mouse models. Moreover, we highlight the significance of model selection in immunotherapy studies, and demonstrate a role for our novel endoscopyguided orthotopic CRC model that could supplement current subcutaneous models to increase the translational potential of preclinical CRC immunotherapeutic studies.

\section{MATERIALS AND METHODS}

\section{CRC cell lines}

CT26, a CRC cell line generated from BABL/C mice, was purchased from the American Type Culture Collection (ATCC, Manassas, VA) and was cultured in RPMI-1640 medium with 10\% fetal bovine serum (FBS) and $1 \%$ penicillin-streptomycin (pen-strep). MC38, a CRC cell line generated from C57BL/6 mice, was a gift from Dr. Nicholas Haining (Harvard University) and was cultured in Dulbecco's modified Eagle's medium (DMEM) with $10 \%$ FBS and 1\% pen-strep. HT29, a human CRC line, was purchased from ATCC and cultured in McCoy's $5 \mathrm{~A}$ medium with $10 \%$ FBS and $1 \%$ pen-strep. For in vivo imaging experiments, we generated a stably transfected CT26 cell line with firefly luciferase (CT26-Luc). Cell lines obtained from ATCC resource were authenticated by the vendor.

\section{Mice}

BALB/c mice (6-8 weeks old, Jackson Laboratory, Bar Harbor, ME) were used for grafts using CT26 and CT26-Luc cells. C57/B6 mice (6-8 weeks old, Charles River Laboratories, Wilmington, MA) were used for MC38 grafts. Athymic nude mice (6-8 weeks old, Charles River Laboratories) were used for HT29 grafts. All mice were kept in a specific pathogen-free facility and had unrestricted access to water and food and a controlled 12-hr day-night cycle. Animal studies were approved by the institutional animal care and use committee of the University of Minnesota.

\section{Tumor implantation}

To perform in vivo high-resolution colonoscopies, we used the Mainz Coloview mini-endoscopic system (Karl Storz Endoskope, Tuttlingen, Germany). For orthotopic tumor cell implantation in the colon wall, we anesthetized mice with ketamine $(100 \mathrm{mg} / \mathrm{kg})$ combined with xylazine $(10 \mathrm{mg} / \mathrm{kg})$ via intraperitoneal injection. To minimize colon movement, contraction, and secretion, we administered atropine $(0.04 \mathrm{mg} / \mathrm{kg}$, intraperitoneally). After tumor cell implantation, mice were put on a heating pad until fully recovered. When monitoring tumors, we anesthetized mice with Avertin (250 to $500 \mathrm{mg} / \mathrm{kg}$, intraperitoneally) 5 minutes before the procedure, to minimize duration of anesthesia. For subcutaneous tumor cell implantation, we suspended tumor cells in a Matrigel matrix, then inoculated the suspension in the flank of legs.

\section{Treatment arm}

After tumor cell implantation, mice were randomly separated into a treatment arm and a control arm. In the treatment arm, mice were injected with anti-mouse PD1 (Clone: RMP1-14, $10 \mathrm{mg} / \mathrm{kg}$, twice per week) and antimouse CLTA4 (Clone: UC10-4F10-11, $5 \mathrm{mg} / \mathrm{kg}$, twice per week) (both from BioCell Technology LLC, Newport Beach, CA). In the control arm, mice were injected with an IgG isotype (15 mg/kg, twice per week) (BioLegend, San Diego, CA).

\section{In vivo imaging}

To monitor orthotopic CRC tumors established by CT26-Luc cells, we used the IVIS Spectrum in vivo imaging system (PerkinElmer, Waltham, MA); 10 minutes before imaging, we injected mice with D-Luciferin, GoldBio $(150 \mathrm{mg} / \mathrm{kg}$, intraperitoneally) and then anesthetized them with isoflurane. For all mice, we set exposure time of imaging as $60 \mathrm{sec}$.

\section{Flow cytometry}

Flow cytometry was used to measure immune cell infiltration and activation markers. Harvested tumor tissue was digested in a solution of collagenase IV $(5 \mathrm{mg} / \mathrm{ml})$ and deoxyribonuclease (DNase, 50 units $/ \mathrm{ml}$ ) at $37^{\circ} \mathrm{C}$ for $1 \mathrm{hr}$ and filtered through a $40-\mu \mathrm{m}$ cell strainer. Cells were centrifuged and resuspended in red blood cell lysis buffer for 10 minutes, followed by another round of centrifugation.

The following antibodies were purchased from BioLegend or BD Biosciences (San Jose, CA): CD3 (17A2), CD19 (6D5), CD4 (GK1.5), CD8 (53-6.7), CD11b (M1/70), CD11c (N418), CD28 (37.51), PD1 (29F.1A12), CTLA4 (UC10-4B9), PDL1 (10F.9G2), CD44 (IM7), CD62L (MEL-14), interferon-gamma (IFN $\gamma$, XMG1.2), Ly6C (HK1.4), Ly6G (1A8), F4/80 (BM8), and I-Ad (AMS-32.1). Cells were stained with surface marker antibodies first, and then fixed and permeabilized for staining with intracellular markers. Data was analyzed using FlowJo software (Tree Star, Inc., Ashland, OR).

\section{Histology and immunostaining}

Immediately after mice were sacrificed, tumor tissue was fixed in $10 \%$ formalin before paraffin embedding. Standard procedures were used for hematoxylin and eosin (H\&E) staining. For immunostaining, formalinfixed, paraffin-embedded tissue was treated with xylene, 
rehydrated with ethanol, and heated in a microwave with citric buffer to retrieve antigens. For blocking purpose, the tissues were incubated for 30 minutes, with $5 \%$ bovine serum albumin buffer. Followed by overnight incubation at $4^{\circ} \mathrm{C}$, with primary antibodies: anti-CD3 antibody, anti-CD4 antibody, and anti-NKp46 antibody (Abcam, Cambridge, United Kingdom) at 1:100 dilutions, anti-CD8 antibody (Novus Biologicals, Minneapolis, MN) at 1:20 dilution, anti-CD31 (Novus Biologicals, Minneapolis, $\mathrm{MN}$ ) at 1:100, anti-F4/80 (R\&D system Minneapolis, $\mathrm{MN}$ ) at 1:100, anti-CD206 (R\&D system Minneapolis, $\mathrm{MN}$ ) at 1:100, as well as anti-CD80 (Novus Biologicals, Minneapolis, MN) at 1:100. After washing, tissues were incubated with fluorescence-conjugated secondary antibodies at room temperature for $1 \mathrm{hr}$. Slides were prepared with antifade mountant with 4',6-diamidino-2phenylindole (DAPI).

\section{RT-qPCR}

The mirVana microRNA (miRNA) Isolation Kit was used to extract RNA. We used 500 ng of total RNA for real-time quantitative reverse-transcriptase polymerase chain reaction (RT-qPCR) analysis with the QuantiTect Reverse Transcription Kit (Qiagen, Hilden, Germany). To measure cDNA samples, we used the LightCycler 480 Instrument (Roche Life Science, Indianapolis, IN) normalized to $18 \mathrm{~S}$ ribosomal RNA (rRNA) expression. Primer sequences are included in Supplementary Table 1.

\section{ELISA}

ELISAs were performed to measure granzyme B, IFN $\gamma$, IL6 and IL2 using ELISA kits from Affymetrix (Santa Clara, CA) according to manufaturer's protocol. All samples were normalized based on protein concentrations measured using a BCA protein assay (Pierce Chemical Company, Dallas, TX).

\section{Statistical analysis}

For all statistical analyses, we used GraphPad Prism 6.0 (GraphPad Software, San Diego, CA). To compare the treatment arm and the control arm, we used the Student $t$ test. For multiple group data, we used the oneway analysis of variance (ANOVA) method; for multiple pairwise comparisons, we performed a Bonferroni post hoc adjustment. All data are plotted as the mean \pm standard deviation (SD). Two-sided $P$ values $<0.05$ were considered statistically significant.

\section{Author contributions}

$\mathrm{XZ}$ and SS contributed to the conception and design of the work; XZ and LL were responsible for data collection; XZ, LL, TS and SS contributed in data analysis and interpretation, drafting the article, and final approval of the version to be published.

\section{ACKNOWLEDGMENTS}

We thank Makayla Maile for helpful discussions and training with the small animal endoscopy. We thank Dr. Mary Knatterud and Leeanne Dongses for assisting in manuscript preparation. We thank Dr. Lalitha Belur and our lab members Dr. Anne Sarver, Ce Yuan and Nidhi Desai for their support during this project.

\section{CONFLICTS OF INTEREST}

The authors report no conflicts of interest.

\section{FUNDING}

This project was supported by startup fund provided to SS by the Department of Surgery, University of Minnesota. TKS is supported by the Mezin-Koats Colon Cancer Research Fund, the Department of Defense (W81XWH-16-1-0427), and the Masonic Cancer Center (NCI/NIH Project grant P30-CA77598). Graduate fellowship of $\mathrm{XZ}$ is supported by the Department of Surgery GI research fellowship.

\section{REFERENCES}

1. Palucka AK, Coussens LM. The basis of oncoimmunology. Cell. 2016; 164:1233-1247.

2. Fesnak AD, June CH, Levine BL. Engineered T cells: the promise and challenges of cancer immunotherapy. Nat Rev Cancer. 2016; 16:566-581.

3. Sharma P, Allison JP. The future of immune checkpoint therapy. Science. 2015; 348:56-61.

4. Borghaei H, Paz-Ares L, Horn L, Spigel DR, Steins M, Ready NE, Chow LQ, Vokes EE, Felip E, Holgado E, Barlesi F, Kohlhaufl M, Arrieta O, et al. Nivolumab versus docetaxel in advanced nonsquamous non-small-cell lung cancer. N Engl J Med. 2015; 373:1627-1639.

5. Motzer RJ, Escudier B, McDermott DF, George S, Hammers HJ, Srinivas S, Tykodi SS, Sosman JA, Procopio G, Plimack ER, Castellano D, Choueiri TK, Gurney H, et al. Nivolumab versus everolimus in advanced renal-cell carcinoma. N Engl J Med. 2015; 373:1803-1813.

6. Robert C, Schachter J, Long GV, Arance A, Grob JJ, Mortier L, Daud A, Carlino MS, McNeil C, Lotem M, Larkin J, Lorigan P, Neyns B, et al. Pembrolizumab versus ipilimumab in advanced melanoma. N Engl J Med. 2015; 372:2521-2532. 
7. Xiao Y, Freeman GJ. The microsatellite instable subset of colorectal cancer is a particularly good candidate for checkpoint blockade immunotherapy. Cancer Discov. 2015; 5:16-18.

8. Jure-Kunkel M, Masters G, Girit E, Dito G, Lee F, Hunt JT, Humphrey R. Synergy between chemotherapeutic agents and CTLA-4 blockade in preclinical tumor models. Cancer Immunol Immunother. 2013; 62:1533-1545.

9. Zhao DX, Li ZJ, Zhang Y, Zhang XN, Zhao KC, Li YG, Zhang MM, Yu XW, Liu MY, Li Y. Enhanced antitumor immunity is elicited by adenovirus-mediated gene transfer of CCL21 and IL-15 in murine colon carcinomas. Cell Immunol. 2014; 289:155-161.

10. Lehmann B, Biburger M, Brückner C, Ipsen-Escobedo A, Gordan S, Lehmann C, Voehringer D, Winkler T, Schaft N, Dudziak D. Tumor location determines tissue-specific recruitment of tumor-associated macrophages and antibodydependent immunotherapy response. Sci Immunol. 2017; 2:eaah6413.

11. Zitvogel L, Pitt JM, Daillere R, Smyth MJ, Kroemer G. Mouse models in oncoimmunology. Nat Rev Cancer. 2016; 16:759-773.

12. Masugi Y, Nishihara R, Yang J, Mima K, da Silva A, Shi Y, Inamura K, Cao Y, Song M, Nowak JA, Liao X, Nosho K, Chan AT, et al. Tumour CD274 (PD-L1) expression and T cells in colorectal cancer. Gut. 2016 May 5. doi: 10.1136/ gutjnl-2016-311421. [Epub ahead of print]

13. Deschoolmeester V, Baay M, Lardon F, Pauwels P, Peeters $\mathrm{M}$. Immune cells in colorectal cancer: prognostic relevance and role of MSI. Cancer Microenviron. 2011; 4:377-392.

14. Selby MJ, Engelhardt JJ, Johnston RJ, Lu LS, Han M, Thudium K, Yao D, Quigley M, Valle J, Wang C, Chen B, Cardarelli PM, Blanset D, Korman AJ. Preclinical development of ipilimumab and nivolumab combination immunotherapy: mouse tumor models, in vitro functional studies, and cynomolgus macaque toxicology. PLoS One. 2016; 11:e0161779.

15. Zhao X, Subramanian S. Intrinsic resistance of solid tumors to immune checkpoint blockade therapy. Cancer Res. 2017; 77:817-822.

16. Bibby MC. Orthotopic models of cancer for preclinical drug evaluation: advantages and disadvantages. Eur J Cancer. 2004; 40:852-857.

17. Mittal VK, Bhullar JS, Jayant K. Animal models of human colorectal cancer: current status, uses and limitations. World J Gastroenterol. 2015; 21:11854-11861.

18. Bettenworth D, Mucke MM, Schwegmann K, Faust A, Poremba C, Schafers M, Domagk D, Lenz P. Endoscopyguided orthotopic implantation of colorectal cancer cells results in metastatic colorectal cancer in mice. Clin Exp Metastasis. 2016; 33:551-562.

19. Fridman WH, Pages F, Sautes-Fridman C, Galon J. The immune contexture in human tumours: impact on clinical outcome. Nat Rev Cancer. 2012; 12:298-306.
20. Beura LK, Hamilton SE, Bi K, Schenkel JM, Odumade OA, Casey KA, Thompson EA, Fraser KA, Rosato PC, Filali-Mouhim A, Sekaly RP, Jenkins MK, Vezys V, et al. Normalizing the environment recapitulates adult human immune traits in laboratory mice. Nature. 2016; 532:512-516.

21. Gould SE, Junttila MR, de Sauvage FJ. Translational value of mouse models in oncology drug development. Nat Med. 2015; 21:431-439.

22. Housseau F, Llosa NJ. Immune checkpoint blockade in microsatellite instable c cancers: back to the clinic. Oncoimmunology. 2015; 4:e1008858.

23. Weide B, Martens A, Hassel JC, Berking C, Postow MA, Bisschop K, Simeone E, Mangana J, Schilling B, Di Giacomo AM, Brenner N, Kahler K, Heinzerling L, et al. Baseline biomarkers for outcome of melanoma patients treated with pembrolizumab. Clin Cancer Res. 2016; 22:5487-5496.

24. Pitt JM, Vetizou M, Daillere R, Roberti MP, Yamazaki T, Routy B, Lepage P, Boneca IG, Chamaillard M, Kroemer G, Zitvogel L. Resistance mechanisms to immune-checkpoint blockade in cancer: tumor-intrinsic and -extrinsic factors. Immunity. 2016; 44:1255-1269.

25. Restifo NP, Smyth MJ, Snyder A. Acquired resistance to immunotherapy and future challenges. Nat Rev Cancer. 2016; 16:121-126.

26. Miller S, Senior PV, Prakash M, Apostolopoulos V, Sakkal S, Nurgali K. Leukocyte populations and IL-6 in the tumor microenvironment of an orthotopic colorectal cancer model. Acta Biochim Biophys Sin (Shanghai). 2016; 48:334-341.

27. Zhao DM, Thornton AM, DiPaolo RJ, Shevach EM. Activated CD4+CD25+ $\mathrm{T}$ cells selectively kill B lymphocytes. Blood. 2006; 107:3925-3932.

28. de Visser KE, Eichten A, Coussens LM. Paradoxical roles of the immune system during cancer development. Nat Rev Cancer. 2006; 6:24-37.

29. Cheng M, Chen Y, Xiao W, Sun R, Tian Z. NK cell-based immunotherapy for malignant diseases. Cell Mol Immunol. 2013; 10:230-252.

30. Schmohl JU, Gleason MK, Dougherty PR, Miller JS, Vallera DA. Heterodimeric bispecific single chain variable fragments ( $\mathrm{scFv}$ ) killer engagers (BiKEs) enhance NK-cell activity against CD133+ colorectal cancer cells. Target Oncol. 2016; 11:353-361.

31. Speroni L, Bustuoabad Vde L, Gasparri J, Chiaramoni NS, Taira MC, Ruggiero RA, Alonso Sdel V. Alternative site of implantation affects tumor malignancy and metastatic potential in mice: its comparison to the flank model. Cancer Biol Ther. 2009; 8:375-379.

32. Nishimura H, Honjo T. PD-1: an inhibitory immunoreceptor involved in peripheral tolerance. Trends Immunol. 2001; 22:265-268.

33. Schwartz RH. Costimulation of T lymphocytes: the role of CD28, CTLA-4, and B7/BB1 in interleukin-2 production and immunotherapy. Cell. 1992; 71:1065-1068. 
34. Pfirschke C, Engblom C, Rickelt S, Cortez-Retamozo V, Garris C, Pucci F, Yamazaki T, Poirier-Colame V, Newton A, Redouane Y, Lin YJ, Wojtkiewicz G, Iwamoto Y, et al. Immunogenic chemotherapy sensitizes tumors to checkpoint blockade therapy. Immunity. 2016; 44:343-354.

35. Moynihan KD, Opel CF, Szeto GL, Tzeng A, Zhu EF, Engreitz JM, Williams RT, Rakhra K, Zhang MH, Rothschilds AM, Kumari S, Kelly RL, Kwan BH, et al. Eradication of large established tumors in mice by combination immunotherapy that engages innate and adaptive immune responses. Nat Med. 2016; 22:1402-1410.

36. Li G, Liu D, Cooper TK, Kimchi ET, Qi X, Avella DM, Li N, Yang QX, Kester M, Rountree CB, Kaifi JT, Cole DJ, Rockey DC, et al. Successful chemoimmunotherapy against hepatocellular cancer in a novel murine model. J Hepatol. 2016; 66:75-85.

37. Curran MA, Montalvo W, Yagita H, Allison JP. PD-1 and CTLA-4 combination blockade expands infiltrating $\mathrm{T}$ cells and reduces regulatory $\mathrm{T}$ and myeloid cells within B16 melanoma tumors. Proc Natl Acad Sci U S A. 2010; 107:4275-4280.

38. Mosely SI, Prime JE, Sainson RC, Koopmann JO, Wang DY, Greenawalt DM, Ahdesmaki MJ, Leyland R, Mullins S, Pacelli L, Marcus D, Anderton J, Watkins A, et al. Rational selection of syngeneic preclinical tumor models for immunotherapeutic drug discovery. Cancer Immunol Res. 2016; 5:29-41. 